\title{
Effects of Oligofructose Enriched Inulin on Protein Bound Uremic Toxin (PBUT) in Dialysis Dependent Chronic Kidney Patients: A Multicentric Indian Clinical Experience
}

\section{Parekh $\mathrm{AD}^{1 *}$, Shah JB${ }^{1}$, Vakil JM${ }^{2}$, Mavani $\mathrm{SB}^{3}$ and Dalal SS}

${ }^{1}$ Sal Hospital, India

${ }^{2}$ Bodyline Hospital, India

${ }^{3}$ Mavani Dialysis \& Kidney Center, India

${ }^{4}$ Sterling Hospital, India

\section{Research Article}

Volume 5 Issue 3

Received Date: September 21, 2020

Published Date: October 05, 2020

DOI: $10.23880 /$ oajun-16000185

*Corresponding author: Apurva D Parekh, Sal Hospital, Ahmedabad, Gujrat-380054, India,

Tel: 9824542389

\section{Abstract}

Monitoring of uremic toxins is a vital tool in diagnostics and treatment of chronic kidney disease (also known as CKD). The gradual loss of renal filtering capacity of kidney is the prime symptom of CKD. Every year millions of patients are facing minor complications related to acute kidney disorders, which gradually give rise to CKD over the time period. The patients that are dialysis-dependent also face various complications related to the removal of uremic toxins, which is associated with renal as well as cardiac complicacy. To prevent progression of cardiovascular toxicity and CKD associated complications, we have tested the effect of oligofructose enriched inulin as a potential candidate to observe the post-consumption effects on the dialysis-dependent patients with confirmed diagnosis of Chronic Kidney Disease.

In a multicentric open label study 2 groups of patients have been studied among which the first group is composed of 10 healthy volunteer candidates named as the control group. The second group is consisting of 73 (excluding the dropouts) dialysis dependent CKD patients with confirmed diagnosis, termed as the treatment group. Fidotox (Manufactured by La Renon Health care Private Limited) was provided as an oral supplement daily for 28 days and the results were compared from day 1 to day 28. To estimate and predict the post-withdrawal efficacy of the inulin containing formulation a follow up assessment was performed on day 90 . The data was subjected to statistics and $p$ values were calculated to observe the potential efficacy of the significance level. It was observed that the oligofructose enriched inulin has the capacity to reduce the protein bound uremic toxins i.e. Indoxyl Sulphate (IS) and p-Cresol Sulphate. The present study concludes that inulin containing formulation is a potential candidate that possibly helps in the addressing the protein bound uremic toxins in dialysis dependent CKD patients.

Keywords: Chronic kidney disorder; p-Cresol Sulphate; Indoxyl Sulphate; Inulin; Renal indication

Abbreviations: CKD: Chronic Kidney Disease; IS: Indoxyl Sulphate; CV: Cardiovascular; pCS: p-Cresyl Sulfate; HD: Hemodialysis.

\section{Introduction}

With rise of CKD patients, renal disorders are among the top 50 health issues in countries like India and China. 


\section{Open Access Journal of Urology \& Nephrology}

Developing countries where the number of elderly people is raising fast, the disappointing number of renal disease patients are also growing fast. Almost $10 \%$ population of the world is suffering from CKD followed by millions of annual deaths. Alone in 2017, 1.2 million deaths were reported from CKD globally (Verma, 2015). CKD is one of the prime non-communicable diseases that are responsible for enhanced morbidity and mortality burden. Its alarming growth indicates better development tools and drugs for the management of CKD and associated risks [1]. Chronic kidney disease is an important issue that exists in front of health care providers in World. The increased prevalence of CKD in our country is unignorable and alarming problem of epidemic proportion in India. India currently has $\sim 1200+$ nephrologist in the country. Of these nephrologists, north, south, west, east and central part of India have 35.5\%, 30\%, $23 \%, 9 \%$ and $2.5 \%$ nephrologist respectively with more than 1500 haemodialysis units $[2,3]$.

There are 2-4 dialysis dependent CKD patients per dialysis facility. In India alone, diabetes and hypertension today account for $40-60 \%$ cases of CKD. Majority of CKD patients also have 10-100 times existing risk of cardiovascular (CV) discomfort. Development of possibly preventive strategies to CKD patients to prevent CVD progression is of great significance [4-6]. Uremic illness is a consequence closely related to form various other associated diseases and clinical conditions. The accumulation of proteolytic substances occurs due to the inadequate removal of protein breakdown products. These biologically active uremic toxins not only responsible to cause renal load in CKD and dialysis patients but they can accumulate into other vital organs such as heart by systemic circulation. This situation is known to enhance the progression of cardiovascular threats to the patient silently.

CKD patients are bound to depend on haemodialysis for the removal of protein metabolites and uremic toxins due to the compromised nephrotic filtration. There is an immediate need to address the development of mitigation strategies to remove the protein bound uremic toxins in dialysis dependent CKD patients. The intake of essential amino acids can't be stopped in any way completely even though the removal of the metabolite products is critically difficult in CKD patients. The proteins taken from dietary sources will have a fate to give rise to various amino acids in vivo. Intestinal microbiota reacts with undigested or other available proteins to give rise to a variety of compounds [7]. The large molecules of the L- tyrosine, and L-phenylalanine give rise to the molecule $\mathrm{p}$ Cresol Sulphate while tryptophan forms the molecules of Indole, which further become more critical protein bound uremic toxin i.e. Indoxyl Sulphate, when bound with sulphate group during glucuronidation in liver $[8,9]$. From liver, it reaches to blood where it binds to proteins and become protein bound uremic toxin. When these proteins bound uremic toxins go into systemic circulation, they accumulate into various organs like heart and lead to vascular calcification and increase cardiovascular mortality and morbidity.

Indoxyl sulfate (IS) and p-cresol, or p-cresyl sulfate (pCS) are potential but relatively less explored uremic toxins that have the recent reports of accumulation in the organs of chronic kidney disease (CKD) patients. The inability of kidney to eliminate uremic toxins from the body causes a threat to existing renal capacity and therefore considered as the adverse implication on CKD patients. Although in the past decade a lot of development has been done in the field of renal replacement techniques for long-term life-saving protocols however the high cost for management forces clinicians to find the new therapeutic option for better management of CKD and dialysis $[10,11]$. Better preservation of residual renal function is an essential strategy for the management of CKD and dialysis-dependent patients to reduce mortality and enhance survival.

The mitigation strategies of IS and PCS include use of gastrointestinal sequestration using oral adsorbents, dietary restrictions and dialysis procedure for renal clearance. Use of pre-biotics, pro-biotics, and synbiotics are efficient management strategies for dialysis dependent CKD patients. The proposed molecule Inulin is an oligosaccharide polymer that is made up of fructose molecules. Several functional and nutritional properties of oligofructose made it an individual for further use as a healthy substance. In inulin, linear fructose polymers and oligomers are each linked by $\beta$ (21) bonds [12]. The good microbiota of the gut is intelligent enough to choose the glucose and similar molecule as a metabolite if available in vivo. Inulin stimulates the growth of intestinal bifidobacteria and it does not contribute to serum glucose enhancement of insulin secretion.

The present study is an effort to examine the role of oligofructose enriched Inulin as a potential therapeutic agent for the management of dialysis dependent CKD patients by accumulated serum IS and pCS mitigation potential analysis to modify the reduced cardiovascular and renal load $[13,14]$.

\section{Methodology}

The objective of the study is to monitor the effects of oligofructose enriched Inulin supplementation on renal health by observing levels of serum Indoxyl Sulfate and p-Cresol in dialysis dependent CKD patients in comparison to healthy individuals. A four-week (28 days) multicentric, open label study was performed to observe the after-effects of "Fidotox" an oligofructose enriched Inulin supplementation manufactured by La Ranon Healthcare Private Limited, 


\section{Open Access Journal of Urology \& Nephrology}

Ahmedabad, India. The written consent from all the patients has been collected and identities were kept confidential to meet ethical principles having their origin in Declaration of Helsinki. Each sachet of Fidotox is consists of $10 \mathrm{gm}$ of Inulin associated with $0.3 \mathrm{~g}$ betaine and $0.1 \mathrm{~g}$ associated sugar and accessory flavouring substances. During the study as per inclusion criterion individual were selected randomly with an age range between 18 to 65 years and with stage 4-5 dialysis dependent CKD patients, without any gender differentiation, with no prior history to bowel obstruction or related disorders. Pregnant ladies and individuals with severe comorbidities were excluded from the study. Without any treatment samples from control, participants were collected to minimize the differences in literature-based reference ranges. The dialysis dependent CKD patients received once daily sachet $(10 \mathrm{gm} /$ day $)$ of Fidotox for initial 7 days orally, diluted with $100 \mathrm{ml}$ of lukewarm water. From the second week, the dose has been increased to twice daily (20gm/day) for the next 3 weeks. The sample for the present study includes 10 subjects for healthy individuals for mean reference range or uremic toxins simultaneously and 73 completed subjects for dialysis dependent CKD patients with the goal of understanding reduction of accumulated serum IS and pCS after 4 weeks treatment.

The initial number of dialysis dependent CKD patients at day 0 was 94 , which reduced to 86 due to 8 patients being lost to follow up on day 28. Day 90 samples were collected for 73 patients to analyse prolonged efficacy of Fidotox. The study samples were analysed statistically for assessment of possible correlation of the clinical study using 73 patients which were consistent through-out. After the 4 weeks (28 days) study a withdrawal follow up was also performed in a similar manner to identify the possible protective outcome of the treatment. Serum Indoxyl Sulfate and p-Cresol Sulphate were determined by enzyme-linked immunosorbent assay (ELISA) test method working on double antibody sandwich enzyme-linked immunosorbent one-step assay. For detection of indoxyl sulphate in patient's serum the FINE TEST ELISA kit manufactured by Wuhan Fine Biotech Co. Limited with a detection range of $31.25 \mathrm{ng} / \mathrm{mL}-2000 \mathrm{ng} / \mathrm{mL}$. For detection of p-cresol sulphate QAYEE BIO ELISA kit was used with a detection range $15.63 \mathrm{pg} / \mathrm{mL}-1000 \mathrm{pg} / \mathrm{mL}$.

\section{Results}

A baseline normal reference range for serum Indoxyl Sulphate and $\mathrm{p}$ Cresol Sulphate was derived post analysis of samples from healthy subjects with no history of renal disease. Mean and Median reference range for Indoxyl Sulphate were $162.759+61.50 \mathrm{ng} / \mathrm{mL}$ and $167.633 \mathrm{ng} / \mathrm{mL}$. Mean and Median reference range for $\mathrm{p}$ Cresol Sulphate were $592.316+74.11$ and $588.18 \mathrm{pg} / \mathrm{mL}$. It was observed that Day 0 mean serum Indoxyl Sulphate concentration for dialysis dependent CKD patients was $409.144 \mathrm{ng} / \mathrm{mL}$, in comparison to healthy individual group where reported mean concentration was $162.759 \mathrm{ng} / \mathrm{mL}$. The overall study reports that after treatment of Fidotox, on Day 28 mean IS concentration was reduced to $316.217 \mathrm{ng} / \mathrm{mL}$ (mean percent change of $22.71 \%$ from Day 0). On Day 90 IS concentration for dialysis dependent CKD patients remains low i.e. 218.695 (mean percent change of $46.55 \%$ from Day 0) (Table 1 \& Figure 1).

\section{Comparative Analysis of Indoxyl Sulphate concentration for pre and post Fidotox Treatment}

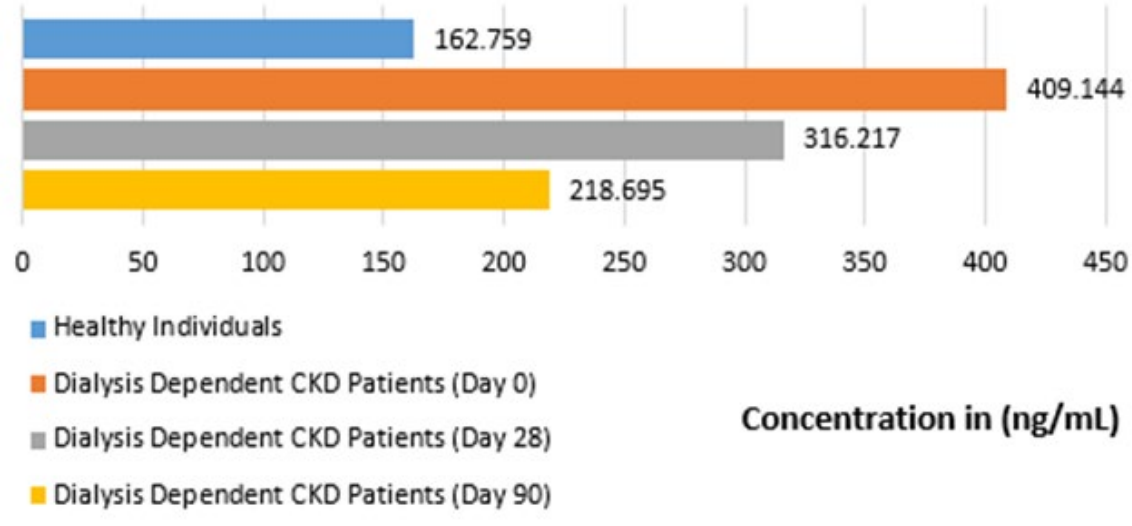

Figure 1: Figure 1 represents the significant $(\mathrm{p}<0.05)$ change in concentration of the Indoxyl Sulfate after 28 days treatment of oral oligofructose enriched inulin supplementation and follow up visit at day 90 among in comparison to the healthy individuals. 


\section{Open Access Journal of Urology \& Nephrology}

\begin{tabular}{|c|c|c|c|c|c|}
\hline S. No. & Day & $\begin{array}{c}\text { Dialysis Dependent CKD } \\
\text { Patients (Mean Value) (ng/ } \\
\text { mL)* }\end{array}$ & $\begin{array}{c}\text { Mean Percentage } \\
\text { Improvement from } \\
\text { Day 0 }\end{array}$ & $\begin{array}{c}\text { Dialysis Dependent } \\
\text { CKD Patients (Median } \\
\text { Value) (ng/ml)** }\end{array}$ & $\begin{array}{c}\text { Median Percentage } \\
\text { Improvement from } \\
\text { Day 0 }\end{array}$ \\
\hline 1 & Day 0 & $409.144 \pm 363.26$ & - & 328.8 & - \\
\hline 2 & Day 28 & $316.217 \pm 200.63$ & $22.71 \%$ & 272.428 & $17.14 \%$ \\
\hline 3 & Day 90 & $218.695 \pm 111.01$ & $46.55 \%$ & 194.72 & $40.78 \%$ \\
\hline
\end{tabular}

${ }^{*}$ Mean Indoxyl Sulphate for Healthy Volunteer $=162.759+61.50$

** Median Indoxyl Sulphate for Healthy Volunteer $=167.633$

Table 1: Table 1 represents the mean \pm Standard deviation and median of the reported concentration of the Indoxyl Sulfate in healthy and oligofructose enriched inulin treated individuals.

It was observed that Day 0 mean serum $\mathrm{p}$ Cresol Sulphate concentration for dialysis dependent CKD patients was $774.566 \mathrm{pg} / \mathrm{mL}$, in comparison to healthy individual group where reported mean concentration was $592.316 \mathrm{pg} / \mathrm{mL}$. The overall study reports that after treatment of Fidotox, on
Day 28 mean pCS concentration was reduced to $472.66 \mathrm{pg} /$ $\mathrm{mL}$ (mean percent change of $38.98 \%$ from Day 0). On Day 90 pCS concentration for dialysis dependent CKD patients remains low i.e. 311.04 (mean percent change of $59.84 \%$ from Day 0) (Table 2 \& Figure 2).

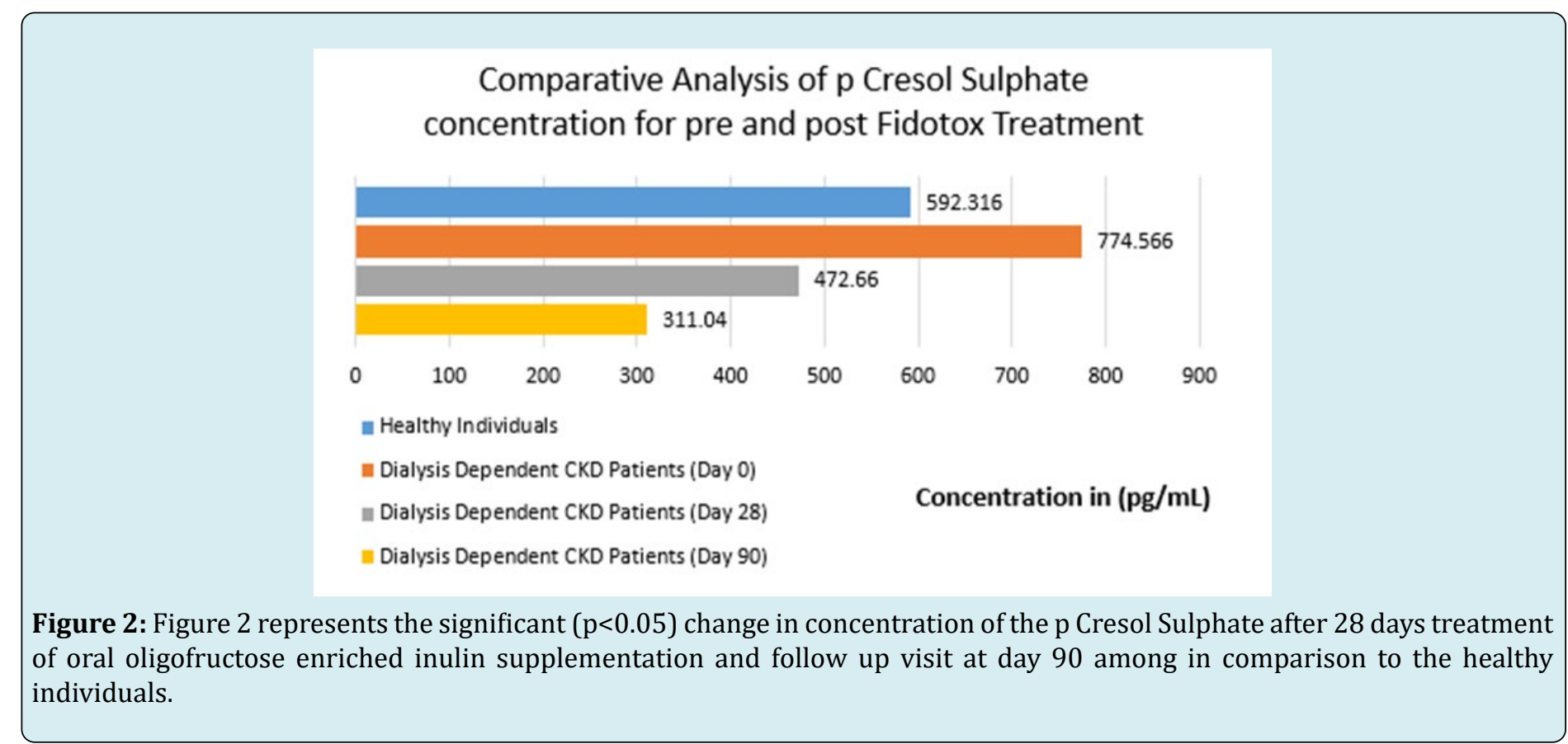

\begin{tabular}{|c|c|c|c|c|c|}
\hline S. No. & Day & $\begin{array}{c}\text { Dialysis Dependent CKD } \\
\text { Patients (Mean Value) (ng/ } \\
\text { mL)* }\end{array}$ & $\begin{array}{c}\text { Mean Percentage } \\
\text { Improvement from } \\
\text { Day 0 }\end{array}$ & $\begin{array}{c}\text { Dialysis Dependent } \\
\text { CKD Patients (Median } \\
\text { Value) (ng/ml)** }\end{array}$ & $\begin{array}{c}\text { Median Percentage } \\
\text { Improvement from } \\
\text { Day 0 }\end{array}$ \\
\hline 1 & Day 0 & $774.566 \pm 267.5$ & - & 790.435 & - \\
\hline 2 & Day 28 & $472.66 \pm 156.79$ & $38.98 \%$ & 445.915 & $43.59 \%$ \\
\hline 3 & Day 90 & $311.04 \pm 266.301$ & $59.84 \%$ & 268.595 & $66.02 \%$ \\
\hline
\end{tabular}

*Mean p Cresol Sulphate for Healthy Volunteer $=592.316+74.11$

$* *$ Median $\mathrm{p}$ Cresol Sulphate for Healthy Volunteer $=588.18$

Table 2: Table 2 represents the mean \pm Standard deviation and median of the reported concentration of the p Cresol Sulfate in healthy and oligofructose enriched inulin treated individuals. 


\section{Open Access Journal of Urology \& Nephrology}

The findings of a randomly selected cohort of healthy as well as dialysis dependent CKD patients of the Indian population indicates the association between oligofructose enriched Inulin intake and serum level of IS and pCS. The reduction in these parameters after the treatment is significant evidence to support the role of Inulin to effectively mediate balance between generation and elimination of uremic toxins. The reduction in these Type III Uremia causing agents are co-metabolites that are well known uremic retention molecules with the potential to enhance the discomfort and life span of dialysis dependent CKD patients [15].

\section{Discussion}

Uremic toxins are biologically active compounds that are made of the result of protein metabolism in vivo. Uraemia is the adverse condition in which is the accumulation of uremic toxins starts affecting the normal renal load and makes the condition adverse for the individual [16]. IS and pCS are the prototype members of the protein-bound uremic toxins. IS is a uremic toxin that is biosynthesized in vivo during tryptophan metabolism from the mediating molecule known as indole. Indole hydroxylation forms the indoxyl in the liver, which further converted into IS by the action of sulfotransferase enzymes. IS (molecular weight of $213.21 \mathrm{~g} /$ mol) has the potential to enhance the renal fibrosis among the cells along with glomerular sclerosis. Proteinuria is one of the symptoms that persist in patients after glomeruli hardening.

Microbial fermentation of L- tyrosine and L-phenylalanine give rise to the molecule pCS in the liver. It is a prototype protein-bound uremic toxin that has a strong association with renal discomfort in CKD patients $[17,9]$. The aromatic pCS formed from sulfation (para-position) of the intestinally generated p-cresol (MW: $108.1 \mathrm{~g} / \mathrm{mol}$ ). $\mathrm{pCS}$ are a high molecular weight of $188.2 \mathrm{~g} / \mathrm{mol}$ compound with high plasma binding affinity (approximately 95\%) to the protein albumin in healthy and CKD patients both [18]. The sulfate form of these uremic toxins is extremely harmful to the body as they don't conveniently eliminate by mechanical dialysis or compensated nephron filtration in renal patients [19].

These uremic toxins transported to the liver for further conjugation with glucuronic acid, under a detoxification and elimination chain. After glucuronidation, these uremic toxins react with available albumin protein and become a high molecular weight conjugate, which is relatively difficult to eliminate in comparison to the original molecule. Due to their physicochemical properties, uremic toxins are one of the protein-bound molecules, which make the status of renal patient critical, if not eliminated on time [20]. In routine healthy individuals, both of these fractions get eliminated by urine, while in CKD patients impaired excretion of these molecules give rise the possible accumulation of these solutes, resulting in higher levels of both conjugates, soluble pCS and bound pCS [21].

Apart from glomerular sclerosis and interstitial renal fibrosis which are caused due to enhanced renal load, IS, and pCS also contribute to the progression of inflammatory reactions, oxidative stress enhancement to create an indirect negative impact on patient's health. In recent literature, the association of uremic toxins has also been studied to cardiovascular functions and related morbidity $[22,23]$.

These toxins travel within the body via soluble mainstream blood and enhance the chances of cardiovascular complications [24]. Due to their protein-bound bulky size, the removal of these molecules becomes difficult with currently available strategies [25]. Existing in the main bloodstream allows these uremic toxins to come in contact with other vital organs such as cardiac machinery and give rise to accessory health implications [26].

In healthy individuals accompanied with routine kidney functions IS and pCS enters the renal tubular cells through organic anion transporters and are subsequently drained into the renal tubules while during chronic renal distress the elimination capacity of these proteins bound uremic toxins diminishes. Consequently, their high concentration further causes increase oxidative stress by free radicals' formation and over-expression of inflammatory genes [27]. A combination of serum indoxyl sulfate with serum proteins affects its elimination by hemodialysis (HD). This situation gives rise to the enhancement of clinical syndromes and cardiovascular diseases and death [28]. In healthy individuals, undetectable IS levels demonstrate the positive correlation between serum IS levels and the severity of kidney disease $[29,30]$.

There are two strategies that could work in the case, one reduced intake of uremic retention molecules, which is partially helpful because the complete restriction of protein is not possible in many cases. The second probability is to manage the uremic fate of these uremic toxins during metabolism, which is done by inulin.

Soluble oligofructose enriched inulin, which is also called fructan also serves as a prebiotic to promote the growth and survival of beneficial gut microbiota. The symbiotic relationship of gut microbial flora is markedly altered during CKD and other renal alterations. The molecular linkage present in this polymer of fructose disable its digestion in the small intestine, thus it reaches the colon and serves as the energy and survival source for beneficial gut bacteria [31]. 


\section{Open Access Journal of Urology \& Nephrology}

The distant colon is the part of the digestive system that plays a vital role in the management of uremic toxin [32]. The involvement of gut microbiota in the management of phenolic compounds is fairly evident in the literature. The incorporation of high fermentable fiber, such as inulin suppresses the proteolytic decomposition and enhances the saccharolytic fermentation indeed. Frequent motion ease and reduced food transit time in the colon also reduce p-cresol biosynthesis [33]. The formation of lactate in the process of metabolism reduces the $\mathrm{pH}$ of colon and at the same time decreases the proteolytic capability that reduces the rate of formation of the molecules required for the progression of uremia. The conversion of inulin into short-chain fatty acids allows the intestine to nourish colon cells and reduce the formation of uremic toxins further.

In connection to the earlier done research present study also conclude that the high intake of fiber was recommended to optimize gastrointestinal function and prevent uremic toxin accumulation in CKD patients, which ultimately results in improvement of additional health benefits along with the declined risk of cardiovascular impairments [34]. The recent findings in support of our study also state that the inulin supplementation in a dose of $10 \mathrm{gm}$ can be beneficial in production of short-chain fatty acids that helps the patient to recover from the uremic toxin production through suppressed protein metabolism [35]. Hence, during the present study, the reduction in the values of uremic toxins in dialysis dependent CKD patients support the positive role of oligosaccharide enriched Inulin in CKD treatment supplementation.

\section{Conclusion}

The present study is first of its kind on Indian population, to show compliance with findings of previous global studies that support the use of present oligofructose enriched inulin for the reduction of higher levels of Indoxyl Sulfate and p-Cresol Sulphate in CKD patients in comparison to healthy individuals. Supplementing the diet of CKD patients with the oligofructose enriched inulin could be an effective method to address the renal load and overall health of CKD patients as it contributes significantly to the reduction of accumulation of p-cresol and Indoxyl Sulfate by enhancing the gut health [36-40]. In light of present findings, fidotox could be advisable for the management of dialysis dependent CKD patients. Meanwhile, a high order molecular study is recommended to elaborate on the molecular mechanism related to the efficacy of the inulin in dialysis CKD patients. The authors also support the need of longer clinical trials with various supporting parameters to determine the clinical usefulness of oligofructose enriched inulin treatment in dialysis dependent CKD patients to delay further renal or cardiovascular deterioration.

\section{References}

1. Agarwal SK, Srivastava RK (2009) Chronic kidney disease in India: challenges and solutions. Nephron Clin Pract 111(3): 197-203.

2. Agarwal SK, Dash SC (2000) Spectrum of renal diseases in India in adults. J Assoc Phy India 48(6): 594-600.

3. Dash SC, Agarwal SK (2006) Incidence of chronic kidney disease in India. Nephrol Dial Transplantation 21(1): 232-233.

4. Langman CB (2006) The epidemic of chronic kidney disease. Med Gen Med 8(3): 55.

5. Tonelli M, Wiebe N, Culleton B, House A, Rabbat C, et al. (2006) Chronic kidney disease and mortality risk: A systematic review. J Am Soc Nephrol 17(7): 2034-2047.

6. (2019) Chronic Kidney Disease in the United States, 2019. Centers for Disease Control and Prevention.

7. Liabeuf S, Drüeke TB, Massy ZA (2011) Protein-bound uremic toxins: new insight from clinical studies. Toxins 3(7): 911-919.

8. Banoglu E, Jha GG, King RS (2001) Hepatic microsomal metabolism of indole to indoxyl, a precursor of indoxyl sulfate. Euro J Drug Metabol Pharmacokin 26(4): 235240.

9. Salmean YA, Segal MS, Palii SP, Dahl WJ (2015) Fiber supplementation lowers plasma p-cresol in chronic kidney disease patients. J Ren Nutr 25(3): 316-320.

10. Varma PP (2015) Prevalence of chronic kidney disease in India-Where are we heading?. Indian J Nephrol 25(3): 133-135.

11. Varughese $S$, Abraham G (2018) A clarion call for change. CJASN 13(5): 802-804.

12. Niness KR (1999) Inulin and oligofructose: what are they?. J Nutr 129(7): 1402-1406.

13. Vanholder R, Bammens B, de Loor H, Glorieux G, Meijers $B$, et al. (2011) Warning: the unfortunate end of p-cresol as a uraemic toxin. Nephrol Dial Transplant 26(5): 14641467.

14. Yang CY, Tarng DC (2018) Diet, gut microbiome and indoxyl sulphate in chronic kidney disease patients. Nephrol 23(4): 16-20.

15. Viaene L, Thijs L, Jin Y, Liu Y, Meijers M, et al. (2014) Heritability and clinical determinants of serum indoxyl sulfate and p-cresyl sulfate, candidate biomarkers of the human microbiome enterotype. PLoS One 9(5): 79682. 


\section{Open Access Journal of Urology \& Nephrology}

16. Watanabe H, Miyamoto $Y$, Honda D, Tanaka H, Wu Q, et al. (2013) p-Cresyl sulfate causes renal tubular cell damage by inducing oxidative stress by activation of NADPH oxidase. Kidney Int 83(4): 582-592.

17. Salmean YA, Segal MS, Henken BL, Canales MT, Zello GA, et al. (2013) Foods with added fiber lower serum creatinine levels in patients with chronic kidney disease. J Ren Nutr 23(2): 29-32.

18. Deltombe O, Van Biesen W, Glorieux G, Massy Z, Dhondt A, et al. (2015) Exploring protein binding of uremic toxins in patients with different stages of chronic kidney disease and during hemodialysis. Toxins (Basel) 7(10): 3933-3946.

19. Radulescu D, Ferechide D (2009) The importance of residual renal function in chronic dialysed patients. J Med Life 2(2): 199-206.

20. Vanholder R, Van Laecke S, Glorieux G (2008) What is new in uremic toxicity?. Pediatr Nephrol 23(8): 12111221.

21. Suchy Dicey AM, Laha T, Hoofnagle A, Newitt R, Sirich LT, et al. (2016) Tubular Secretion in CKD. J Am Soc Nephrol 27(7): 2148-2155.

22. Ito S, Yoshida M (2014) Protein-bound uremic toxins: new culprits of cardiovascular events in chronic kidney disease patients. Toxins (Basel) 6(2): 665-678.

23. Liu WC, Tomino Y, Lu KC (2018) Impacts of indoxyl sulfate and p-cresol sulfate on chronic kidney disease and mitigating effects of AST-120. Toxins (Basel) 10(9): 367.

24. Moradi H, Sica DA, Kalantar Zadeh K (2013) Cardiovascular burden associated with uremic toxins in patients with chronic kidney disease. Am J Nephrol 38(2): 136-148.

25. Lesaffer G, De SR, Lameire N, Dhondt A, Duym P, et al. (2000) Intradialytic removal of protein-bound uraemic toxins: role of solute characteristics and of dialyser membrane. Nephrol Dial Transplant 15(1): 50-57.

26. Lisowska Myjak B (2014) Uremic toxins and their effects on multiple organ systems. Nephron Clin Pract 128(3-4): 303-311.

27. Sun CY, Hsu HH, Wu MS (2013) p-Cresol sulfate and indoxyl sulfate induce similar cellular inflammatory gene expressions in cultured proximal renal tubular cells. Nephrol Dial Transplant 28(1): 70-78.

28. Meijers BK, Claes K, Bammens B, de Loor H, Viaene L, et al. (2010) p-Cresol and cardiovascular risk in mildto-moderate kidney disease. Clin J Am Soc Nephrol 5(7):
1182-1189.

29. Niwa T, Ise MJ (1994) Indoxyl sulfate, a circulating uremic toxin, stimulates the progression of glomerular sclerosis. Lab Clin Med 124(1): 96-104.

30. Duranton F, Cohen G, De Smet R, Rodriguez M, Jankowski J, et al. (2012) Normal and pathologic concentrations of uremic toxins., European Uremic Toxin Work Group. J Am Soc Nephrol 23(7): 1258-1270.

31. Nallu A, Sharma S, Ramezani A, Muralidharan J, Raj D (2017) Gut microbiome in CKD: Challenges and opportunities. Transl Res 179: 24-37.

32. Aronov PA, Luo FJ, Plummer NS, Quan Z, Holmes S, et al. (2011) Colonic contribution to uremic solutes. J Am Soc Nephrol 22(9): 1769-1776.

33. Den Hond E, Geypens B, Ghoos Y (2000) Effect of high performance chicory inulin on constipation. Nutr Res 20(5): 731-736.

34. Wu M, Cai X, Lin J, Zhang X, Scott EM, et al. (2019) Association between fibre intake and indoxyl sulphate/ P-cresyl sulphate in patients with chronic kidney disease: Meta-analysis and systematic review of experimental studies. Clin Nutr 38(5): 2016-2022.

35. O'Connor S, Chouinard-Castonguay S, Gagnon C, Rudkowska I (2017) Prebiotics in the management of components of the metabolic syndrome. Maturitas 104: 11-18.

36. Bikbov Boris (2017) Global, regional, and national burden of chronic kidney disease, 1990-2017: a systematic analysis for the global burden of disease study. The Lancet 395(10225): 709-733.

37. Bres E, Koppe L (2019) Is there still a place for prebiotics in chronic kidney disease?. Nephrol Dial Transplant. 34(11): 18121-1816.

38. Lau WL, Savoj J, Nakata MB, Vaziri ND (2018) Altered microbiome in chronic kidney disease: systemic effects of gut-derived uremic toxins. Clin Sci 132(5): 509-522.

39. Meyer TW, Hostetter TH (2012) Uremic solutes from colon microbes. Kidney Int 81(10): 949-954.

40. Moradi H, Sica DA, Kalantar-Zadeh K (2013) Cardiovascular Burden Associated with Uremic Toxins in Patients with Chronic Kidney Disease. Am J Nephrol 38(2): 136-148. 